\title{
Adenoid cystic carcinoma of the sublingual gland: A case report
}

\author{
Ji-Young Song ${ }^{1, *}$ \\ ${ }^{1}$ Department of Oral and Maxillofacial Surgery, School of Medicine, Jeju National University, Jeju, Korea
}

\section{ABSTRACT}

\begin{abstract}
Adenoid cystic carcinoma (ACC) of the sublingual gland is an extremely rare neoplasm. The clinicopathological characteristics of ACC are slow-growing swelling with or without ulceration, perineural spread, local recurrence, and distant metastasis. This report describes a 58-year-old male who had a slowly growing swelling without ulceration on the right side of the mouth floor that had been present for 1 month. In a radiological examination, the mass showed multilocular cystic features and no bony or tongue muscle invasion. No enlarged cervical lymph nodes were detected. Excisional biopsy and histological analysis showed that the lesion was ACC. In addition to reporting a rare case of ACC, this report also discusses the differential diagnosis and treatment of ACC with a review of the relevant literature. (Imaging Sci Dent 2016; 46: 291-6)
\end{abstract}

KEY WORDS: Carcinoma, Adenoid Cystic; Salivary Gland; Mouth Floor

Adenoid cystic carcinoma (ACC) is a salivary gland malignancy that is an extremely rare neoplasm $(<1 \%$ of all carcinomas of the head and neck area). ${ }^{1}$ It occurs in the submandibular gland in $15 \%-30 \%$ of all cases, in the minor salivary gland in $30 \%$ of cases, and in the parotid gland in $2 \%-15 \%$ of cases. ${ }^{2}$ It frequently occurs between the fifth and sixth decades of life, predominantly in females. $^{3}$

The clinicopathological characteristics of ACC are slowgrowing swelling, locally invasive growth with perineural spread, local recurrence, and distant metastasis to the lung and bone. ${ }^{3,4}$ Metastasis generally occurs via the lymphatics rather than via the blood. ${ }^{5}$

The lesion is generally irregular in shape with bone destruction in computed tomography (CT) imaging and high signal intensity or heterogeneous density in magnetic resonance imaging (MRI). ${ }^{3}$ Radical surgery, radiotherapy, chemotherapy, and concurrent chemoradiotherapy are possible treatment modalities for ACC. The most common technique is radical surgery combined with adjuvant

Received April 21, 2016; Revised July 13, 2016; Accepted July 27, 2016 *Correspondence to : Prof. Ji-Young Song

Department of Oral and Maxillofacial Surgery, School of Medicine, Jeju National University Hospital, Ara 1-dong, Jeju-si, Jeju Special Self-Governing Province 63241, Korea

Tel) 82-64-717-1845,Fax) 82-64-717-1102,E-mail)2460song@naver.com radiotherapy. ${ }^{3-6}$

In this report, we describe a rare case of sublingual gland ACC that was initially misdiagnosed as pleomorphic adenoma on the basis of fine-needle aspiration biopsy, and discuss the differential diagnosis and treatment of ACC with a review of the relevant literature.

\section{Case Report}

A 58-year-old male patient was referred to the oral and

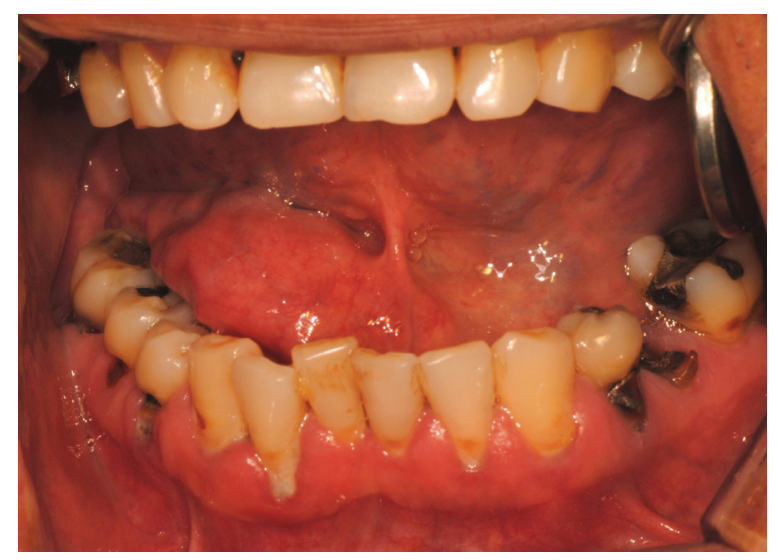

Fig. 1. A mass on the right mouth floor is shown in a clinical photograph. 

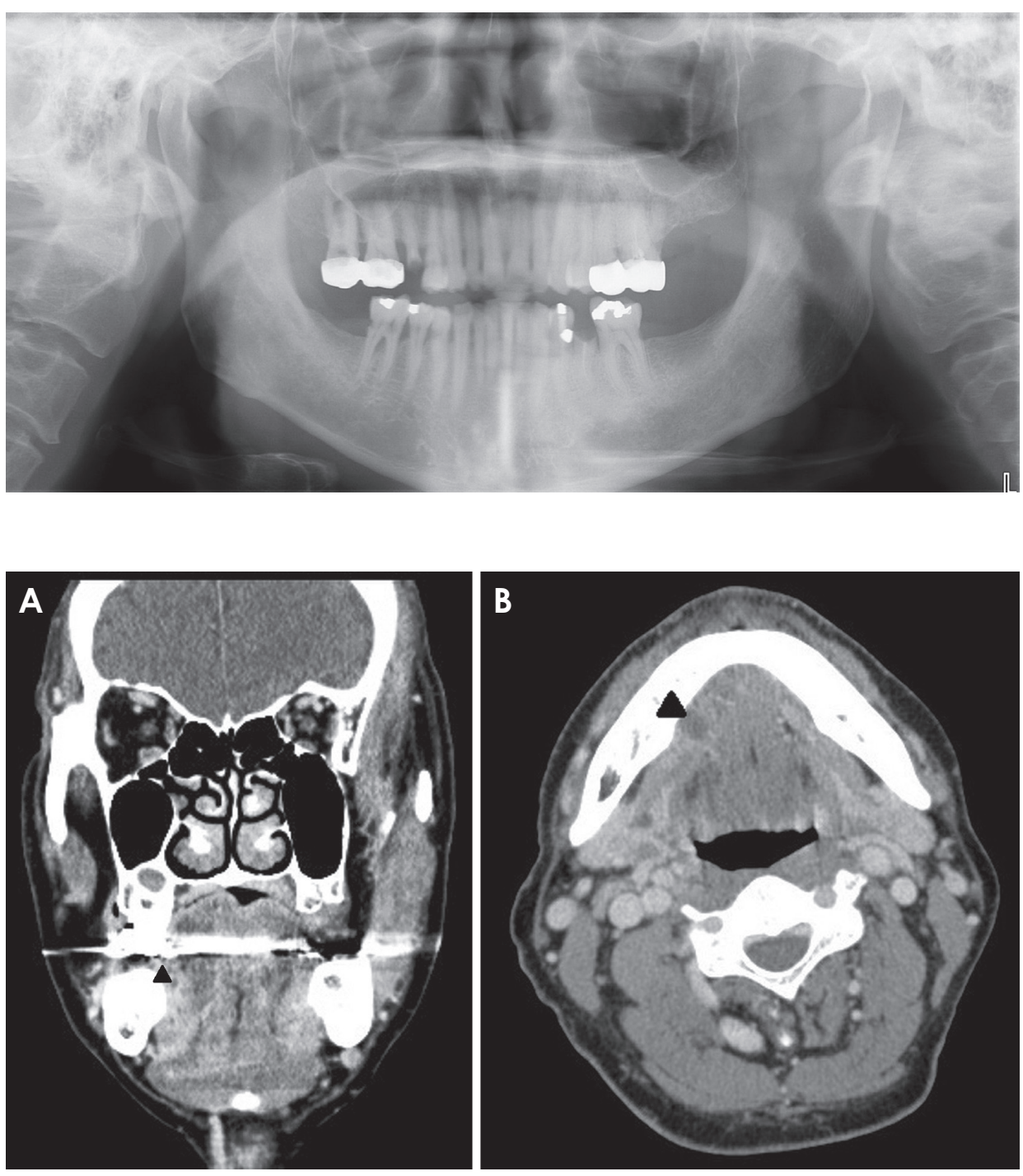

Fig. 2. A dental panoramic image shows no specific findings.

Fig. 3. A. Dilatation of the submandibular gland duct (arrowhead) is shown on a contrast-enhanced computed tomograph (coronal view). B. Cystic density with marginal enhancement in the right sublingual space (arrowhead) is shown on a contrast-enhanced computed tomograph (axial view). maxillofacial surgery department of our institution for the treatment of a slowly growing swelling on the right side of the mouth floor that had been present for 1 month. He had slight dull pain during eating and speaking. The patient stated that the size of the mass changed several times a day. He had a history of smoking and alcohol consumption for more than 30 years. No other specific medical problems were detected, and he had no history of traumatic injury, invasive surgery, bleeding, or discharge. He had no familial history of cancer.

Intraoral examination showed that the mass (approximately $3 \mathrm{~cm}$ in length and $2 \mathrm{~cm}$ in width) was covered with intact mucosa without ulceration. The adjacent teeth and gingiva showed no abnormalities (Fig. 1). In a dental panoramic view, only chronic periodontitis and several retained dental roots were found. No bony irregularities or sialoliths were found (Fig. 2).
Contrast-enhanced CT revealed dilatation of the right submandibular gland duct (Fig. 3A) and a few focal cystic densities in the right sublingual space (Fig. 3B). No bony destruction of the mandible or submandibular stones were detected.

Axially sectioned T1-weighted MRI showed a multilocular cystic mass with heterogeneous intensity and marked peripheral enhancement in the right sublingual space (Fig. 4A). The lesion extended to the mandibular midline anteriorly and to the molar teeth posteriorly. The mass compressed the sublingual gland laterally. The lesion was located superior to the mylohyoid muscle on coronally sectioned T1-weighted MRI (Fig. 4B). Axially sectioned T2-weighted MRI showed a well-defined isointense signal in two-thirds of the anterior part of the mass and heterogeneous intensity in one-third of the posterior part (the remaining third) of the mass (Fig. 4C). Coronally 

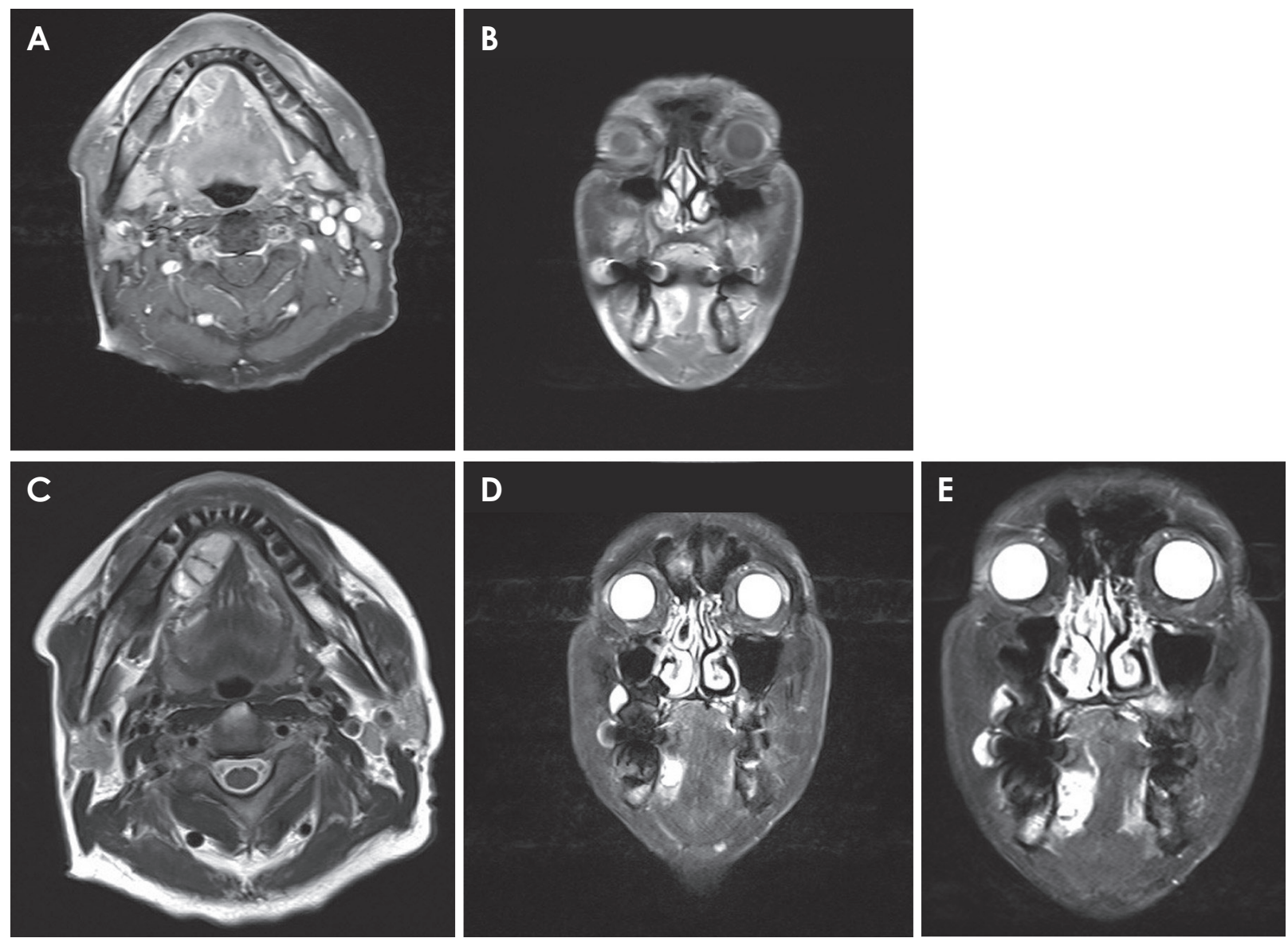

Fig. 4. A. The mass shows heterogeneous intensity with peripheral enhancement in gadolinium-enhanced T1-weighted magnetic resonance imaging (axial view). B. The mass is located superior to the mylohyoid muscle in gadolinium-enhanced T1-weighted magnetic resonance imaging (coronal view). C. Well-defined multilocular cystic mass in the right sublingual space is demonstrated in fat-suppressed T2-weighted magnetic resonance imaging (axial view). D. The mass is surrounded by a hypointense signal, which was suspected to represent a fibrous capsule, in fat-suppressed T2-weighted magnetic resonance imaging (coronal view). E. The location and size of the mass is clearly shown in fat-suppressed T2-weighted magnetic resonance imaging (coronal view).

sectioned T2-weighted MRI showed the mass to be surrounded by a hypointense signal, which was suspected to represent a fibrous capsule surrounding the mass (Fig. 4D). The approximate size of the lesion was $2.6 \mathrm{~cm} \times 1.5$ $\mathrm{cm} \times 2.4 \mathrm{~cm}$ (length $\times$ width $\times$ height) (Fig. 4E). No invasion of the bone or the tongue muscle was found, and the mouth floor was intact. No significantly enlarged lymph nodes were found.

The mass had a well-defined multilocular cystic configuration, and fine-needle aspiration biopsy (FNAB) was indicative of pleomorphic adenoma; therefore, the presurgical diagnosis was pleomorphic adenoma.

Surgery for excision of the mass was accomplished under general anesthesia using laryngoscopy with orotracheal intubation. An incision on the right side of the mouth floor was made and the mass and sublingual gland were resected with preservation of the submandibular gland and lingual nerve. The mass was encapsulated in fibrotic soft tissue and filled with uniform material (Fig. 5). Postoperative pain medications and antibiotics were prescribed for 5 days.

Histological analysis of the excised mass revealed ACC (Fig. 6). After the final diagnosis, an additional resection of the tissue surrounding the resected mass and sublingual gland was performed under general anesthesia. Frozen biopsies performed during surgery were all negative (no malignancy). Additionally, no invasion of the bone and no significantly enlarged lymph nodes were found. Therefore, additional radiotherapy was not considered. The patient has been encouraged to undergo regular check-ups.

\section{Discussion}

ACC is a rare malignancy, and approximately $10 \%$ of 


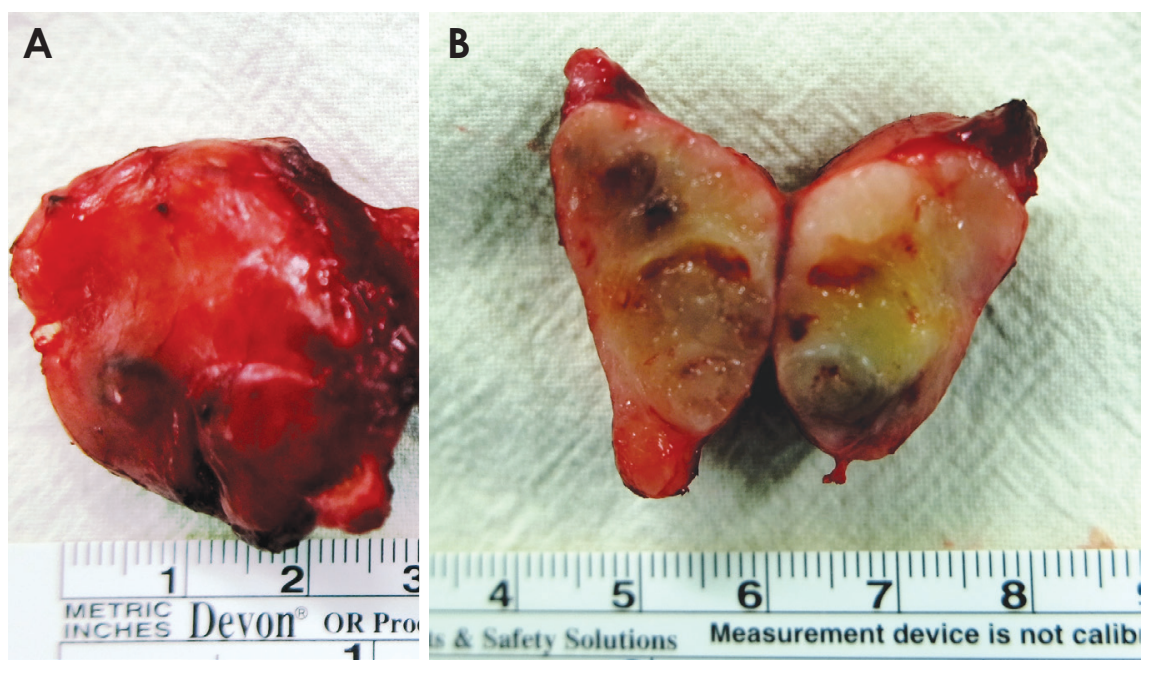

Fig. 5. A. The resected mass is shown to be well capsulated in fibrotic tissue in an intraoperative clinical photograph. B. The resected mass is filled with a uniform material.
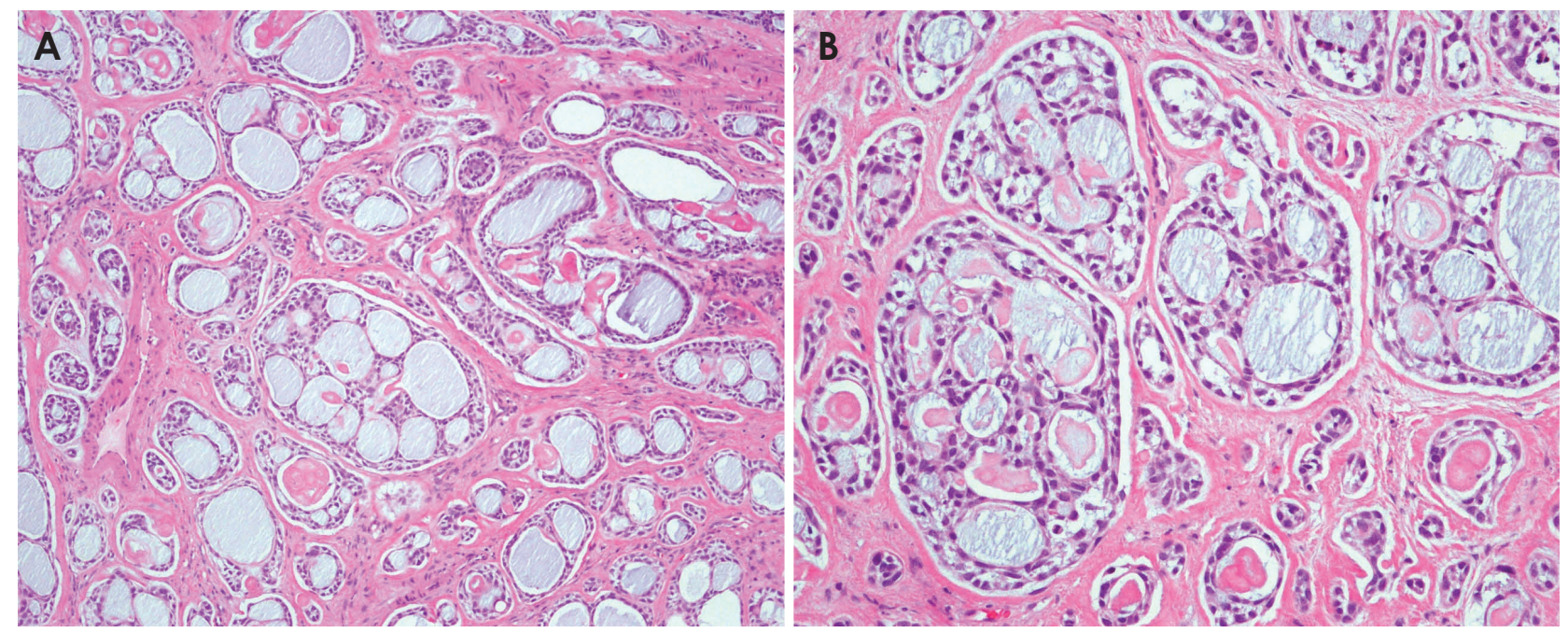

Fig. 6. The histological analysis reveals a typical cribriform adenoid cystic carcinoma (H\&E stain, A. $\times 100$, B. $\times 200)$.

ACC cases occur in the salivary glands, among which ACC of the minor salivary gland has the worst prognosis. $^{7,8}$

The overall 5-year survival rate of patients with head and neck ACC has been reported to be approximately $90.3 \% .^{3}$ In histological analyses, ACC has 3 morphologic patterns: tubular, cribriform, and solid. The tubular and cribriform patterns indicate low-grade tumors, whereas the solid pattern indicates high-grade tumors. ${ }^{9,10}$

Imaging modalities for evaluating the invasion of the perineural, vascular, or skull base regions by ACC include ultrasonography, CT, and MRI. ACC has an echo-free cystic area on ultrasonography. ${ }^{10,11}$ However, ultrasonography is insufficient for evaluating deep neck lesions. The most adequate method for the diagnosis of ACC is MRI, which is more accurate than $\mathrm{CT}$ and ultrasonography. ${ }^{12} \mathrm{ACC}$ can be efficiently evaluated with fat-suppressed $\mathrm{T} 2$-weighted MRI, in which the intensity of the lesion differs according to the histological type of ACC: the solid type with poor prognosis has a low signal intensity, whereas the cribriform and tubular types with better prognoses have a high signal intensity. ${ }^{11,12}$ In our patient, the lesion showed multilocular cystic features with generally high signal intensity on MRI (Fig. 4), and the histological analysis revealed cribriform ACC.

Generally, an irregular margin is found in high-grade salivary gland malignancies, whereas a well-defined margin is found in low-grade salivary malignancies and benign tumors. ${ }^{7,13-15}$ In our case, the lesion had a well-defined margin on MRI, and the histological analysis revealed low- 
grade ACC.

In addition, most salivary gland malignancies have no capsules that are detectable by MRI ${ }^{16,17}$ However, in our case, the mass was enveloped in a fibrous capsule. The capsule might have originated from a dilated submandibular duct, as in a previously reported case. ${ }^{16}$

The differential diagnosis of lesions originating from the sublingual glands includes pleomorphic adenoma, acinic cell carcinoma, and mucoepidermoid carcinoma. ${ }^{18,19}$ Pleomorphic adenomas are the most common benign tumor in the salivary glands. ${ }^{19}$ In MRI findings, pleomorphic adenomas are well defined, have a lobular or spherical shape, and appear as homogeneous-density masses. In cases of hemorrhage, cystic changes, or calcification, the lesions might be heterogeneous. ${ }^{20}$ Acinic cell carcinoma is the second most frequent malignancy in the parotid gland. Mucoepidermoid carcinoma originates from the ductal epithelium. ${ }^{19,20}$ In CT and MRI, acinic cell carcinoma and mucoepidermoid carcinoma have no specific features and are similar to low-grade pleomorphic adenoma. Perineural invasion (fatty changes in the neural foramen and nodular enhancement with a thickened nerve) is detected by CT and MRI in high-grade tumors. ${ }^{10,19-23}$ Distinguishing between benign tumors and malignancies in the sublingual gland using only conventional radiological features is difficult. However, new MRI techniques, such as dynamic contrast-enhanced MRI, diffusion-weighted MRI, or proton magnetic resonance spectroscopy may be helpful. ${ }^{24}$ In dynamic contrast-enhanced MRI, the time-intensity curves (TICs) of malignant tumors show a rapid increase immediately after contrast injection, followed by a gradual decrease, whereas the TIC of benign tumors tends to show a gradual increase. ${ }^{25}$ In diffusion-weighted MRI, the apparent diffusion coefficients (ADCs; the mean of 3-dimensional measurements of water diffusion) of malignant tumors are lower than the ADCs of benign tumors. ${ }^{26}$ In proton magnetic resonance spectroscopy, malignant tumors have lower choline-to-creatine ratios at an echo time of $136 \mathrm{~ms}$ than benign tumors. ${ }^{27}$ Additionally, the differential diagnosis of lesions in the mouth floor includes benign tumors such as lipomas, irritation fibromas, salivary gland lesions (mucoceles and mixed tumors); malignant tumors; and angiomatous lesions. ${ }^{28-30}$ Lipomas are well defined in clinical and radiological examinations. They have a soft feel and a unique yellowish color in clinical examinations and high signal intensity with a well-circumscribed mass on T1-weighted MRI. ${ }^{28}$

Mucocele is associated with a local trauma or rupture of the salivary gland duct and also appears as a well-de- fined cystic lesion with high signal intensity in MRI. ${ }^{29,30}$ Incisional and excisional biopsies assist in the differential diagnosis of these mixed and malignant tumors. ${ }^{28-30}$

In conclusion, masses originating from sublingual glands are rare. FNAB results in false diagnoses such as ACC or pleomorphic adenoma in $33 \%$ of cases, ${ }^{18}$ as occurred in our patient. Therefore, using additional diagnostic tools during the presurgical period, such as incisional biopsy or additional MRI techniques, as well as frozen sectional biopsy during surgery, is helpful for the differential diagnosis and the development of additional treatment plans.

\section{References}

1. Dong J, Tian L, Li S, Mo Y, Liu L, Zhong R. Differences in extension patterns between adenoid cystic carcinoma of the nasopharynx and nasopharyngeal carcinoma on MRI. Int J Clin Exp Pathol 2015; 8: 15960-8.

2. Khan S, Agwani K, Bhargava P, Kumar SP. Adenoid cystic carcinoma presenting as an ulcer on the floor of the mouth: a rare case report. J Korean Assoc Oral Maxillofac Surg 2014; 40: 253-7.

3. Sepulveda I, Platin E, Delgado C, Rojas P. Sinonasal adenoid cystic carcinoma with intracranial invasion and perineural spread: a case report and review of the literature. J Clin Imaging Sci 2015; 5: 57.

4. Feng H, Wang J, Guo P, Xu J, Feng J. C3 vertebral metastases from tongue adenoid cystic carcinoma: a rare case report. Medicine (Baltimore) 2015; 94: e1135.

5. Mesolella M, Luce A, Marino A, Caraglia M, Ricciardiello F, Iengo M. Treatment of c-kit positive adenoid cystic carcinoma of the tongue: a case report. Oncol Lett 2014; 8: 309-12.

6. Triantafillidou K, Dimitrakopoulos J, Iordanidis F, Koufogiannis D. Management of adenoid cystic carcinoma of minor salivary glands. J Oral Maxillofac Surg 2006; 64: 1114-20.

7. Falk GA, El-Hayek K, Morris-Stiff G, Tuthill RJ, Winans CG. Adenoid cystic carcinoma of the base of the tongue: late metastasis to the pancreas. Int J Surg Case Rep 2011; 2: 1-3.

8. Sato K, Ueda Y, Sakurai A, Ishikawa Y, Kaji S, Nojima T, et al. Adenoid cystic carcinoma of the maxillary sinus with gradual histologic transformation to high-grade adenocarcinoma: a comparative report with dedifferentiated carcinoma. Virchows Arch 2006; 448: 204-8.

9. Benson BW. Salivary gland radiology. In: White SC, Pharoah MJ. Oral radiology: principles and interpretation. 5th ed. St. Louis, Mo: Mosby; 2004. p. 658-76.

10. Yousem DM, Kraut MA, Chalian AA. Major salivary gland imaging. Radiology 2000; 216: 19-29.

11. Kumar VS, Prathi VS, Manne RK, Beeraka S, Natarajan K. Adenoid cystic carcinoma of sublingual salivary gland obstructing the submandibular salivary gland duct. J Clin Imaging Sci 2013; 3 (Suppl 1): 10.

12. Ariji Y, Gotoh M, Naitoh M, Izumi M, Shimozato K, Kurita $\mathrm{K}$, et al. Magnetic resonance imaging assessment of tumorous lesions in the floor of the mouth: case reports and review of 
the literature. Oral Radiol 2006; 22: 18-26.

13. Freling NJ, Molenaar WM, Vermey A, Mooyaart EL, Panders AK, Annyas AA, et al. Malignant parotid tumors: clinical use of MR imaging and histologic correlation. Radiology 1992; 185: 691-6.

14. Vogl TJ, Dresel SH, Spath M, Grevers G, Wilimzig C, Schedel HK, et al. Parotid gland: plain and gadolinium-enhanced MR imaging. Radiology 1990; 177: 667-74.

15. Son PM, Brandwein MS. Adenoid cystic carcinoma. In: Som PM, Curtin HD. Head and neck imaging. 4th ed. St. Louis, Mo: Mosby; 2003. p. 2090-6.

16. Saito M, Nishiyama H, Maruyama S, Oda Y, Saku T, Hayashi T. Adenoid cystic carcinoma of sublingual gland involving the submandibular duct. Dentomaxillofac Radiol 2008; 37: 421-4.

17. Huang TT, Chou YF, Wen YH, Chen PR. Resected tumours of the sublingual gland: 15 years' experience. Br J Oral Maxillofac Surg 2016; 54: 625-8.

18. Peravali RK, Bhat HH, Upadya VH, Agarwal A, Naag S. Salivary gland tumors: a diagnostic dilemma! J Maxillofac Oral Surg 2015; 14 (Suppl 1): 438-42.

19. Madani G, Beale T. Tumors of the salivary glands. Semin Ultrasound CT MRI 2006; 27: 452-64.

20. Lee YY, Wong KT, King AD, Ahuja AT. Imaging of salivary gland tumours. Eur J Radiol 2008; 66: 419-36.

21. Ginsberg LE. Imaging of perineural tumor spread in head and neck cancer. Semin Ultrasound CT MR 1999; 20: 175-86.

22. Laccourreye O, Bely N, Halimi P, Guimaraes R, Brasnu D. Cavernous sinus involvement from recurrent adenoid cystic carcinoma. Ann Otol Rhinol Laryngol 1994; 103: 822-5.

23. Curtin HD. Detection of perineural spread: fat is a friend. AJNR Am J Neuroradiol 1998; 19: 1385-6.

24. Swartz JD, Rothman MI, Marlowe FI, Berger AS. MR imaging of parotid mass lesions: attempts at histopathologic differentiation. J Comput Assist Tomogr 1989; 13: 789-96.

25. Assili S, Fathi Kazerooni A, Aghaghazvini L, Saligheh Rad HR, Pirayesh Islamian J. Dynamic contrast magnetic resonance imaging (DCE-MRI) and diffusion weighted MR imaging (DWI) for differentiation between benign and malignant salivary gland tumors. J Biomed Phys Eng 2015; 5: 157-68.

26. Wang J, Takashima S, Takayama F, Kawakami S, Saito A, Matsushita T, et al. Head and neck lesions: characterization with diffusion-weighted echo-planar MR imaging. Radiology 2001; 220: 621-30.

27. King AD, Yeung DK, Ahuja AT, Tse GM, Yuen HY, Wong KT, et al. Salivary gland tumors at in vivo proton MR spectroscopy. Radiology 2005; 237: 563-9.

28. Hoseini AT, Razavi SM, Khabazian A. Lipoma in oral mucosa: two case reports. Dent Res J (Isfahan) 2010; 7: 41-3.

29. Nilesh K, Malik NA, Patil P, Chapi MD. Large plunging ranula presenting as isolated neck swelling: steps in diagnosis and surgical steps in management. J Clin Diagn Res 2015; 9: MD01-3.

30. More CB, Bhavsar K, Varma S, Tailor M. Oral mucocele: a clinical and histopathological study. J Oral Maxillofac Pathol 2014; 18 (Suppl 1): S72-7. 